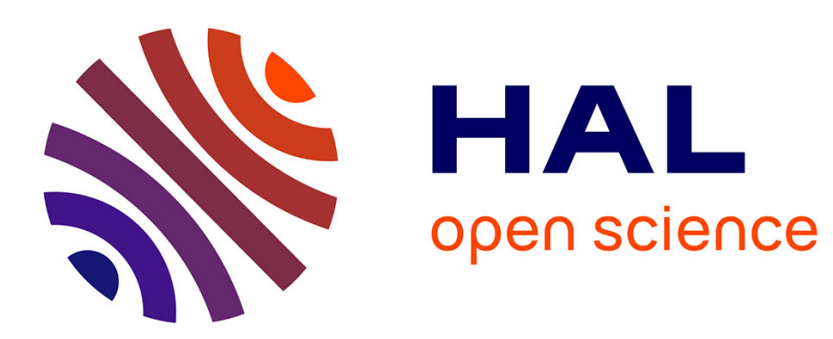

\title{
High frequency vibroacoustics: A radiative transfer equation and radiosity based approach
}

\author{
Alain Le Bot, Emeline Sadoulet-Reboul
}

\section{To cite this version:}

Alain Le Bot, Emeline Sadoulet-Reboul. High frequency vibroacoustics: A radiative transfer equation and radiosity based approach. Wave Motion, 2014, 51, pp.598 - 605. 10.1016/j.wavemoti.2014.01.003 . hal-01073845

\section{HAL Id: hal-01073845 \\ https://hal.science/hal-01073845}

Submitted on 10 Oct 2014

HAL is a multi-disciplinary open access archive for the deposit and dissemination of scientific research documents, whether they are published or not. The documents may come from teaching and research institutions in France or abroad, or from public or private research centers.
L'archive ouverte pluridisciplinaire HAL, est destinée au dépôt et à la diffusion de documents scientifiques de niveau recherche, publiés ou non, émanant des établissements d'enseignement et de recherche français ou étrangers, des laboratoires publics ou privés. 


\title{
High frequency vibroacoustics: A radiative transfer equation and radiosity based approach
}

\author{
A. Le Bot ${ }^{\mathrm{a}}$, E. Sadoulet-Reboul ${ }^{\mathrm{b}}$ \\ ${ }^{a}$ Laboratoire de tribologie et dynamique des systèmes, UMR CNRS 5513, École centrale \\ de Lyon, 36, av. Guy de Collongue 69134 Ecully, France \\ ${ }^{b}$ Institut FEMTO-ST, UMR CNRS 6174, Département de mécanique appliquée, \\ Université de Franche-Comté, 24 rue de l'Epitaphe, F-25000 Besançon, France
}

\begin{abstract}
This paper is a review of the theoretical framework for application of radiative transfer equations to structural dynamics and acoustics. It is shown that under the assumption of geometrical acoustics and when the phase of rays is neglected, a representation of sound field in terms of incoherent fictitious sources provides a sufficiently large framework to embody diffuse as well as specular reflection, steady-state or transient phenomena and even diffraction. Two special cases are mentioned. The so-called radiosity method in acoustics corresponds to a purely diffusing boundary and statistical energy analysis when the sound field is diffuse.
\end{abstract}

Keywords: High-frequency, geometrical acoustics, statistical energy analysis, radiosity, specular reflection, multiple diffraction

\section{Introduction}

The interest of the scientific community in high frequency methods applied to vibro-acoustics is relatively recent compared to other fields of physics such as electrodynamics or acoustics. It seems that ray methods have not been so popular in structural dynamics where most engineers prefer to use softwares based on the governing equations solved by the finite element method.

Email addresses: alain.le-bot@ec-lyon.fr (A. Le Bot), emeline.sadoulet-reboul@univ-fcomte.fr (E. Sadoulet-Reboul) 
However, this 'brute force' approach of vibro-acoustics rapidly encounters a practical limitation. When the frequency increases, the number of natural modes increases. Even for a numerical model of order of tens of millions of degrees of freedom, a reasonable task on current computers, the maximum analysis frequency is still too low and does not permit to cover the entire audio frequency band. This limitation is particularly unfavorable in the automotive industry or for larger structures such as aircraft or launchers which may contain millions of natural modes within the audio frequency band [1]. Furthermore, it might be emphasized that the sensitivity of these computations is poor which raises the question of how useful it is to know the 'exact' sequence of natural modes for systems having a large number of modes.

For these reasons, statistical energy analysis [2] (SEA) has been developed for fifty years. Introduced early in the sixties by R.H. Lyon [3] and many other authors $[4,5,6]$, this theory is based on statistical concepts and applies when sources are random, uncorrelated and when the number of modes is large enough to be considered as a statistical population. Then under certain circumstances [7], sound/vibration reaches a state of diffuse field and equipartition of modal energy (an interesting method to dispense with equipartition is proposed in Refs. $[8,9])$. Then, a simple law emerges: the energy exchange between two coupled subsystems is proportional to the difference in their modal energies. This has served as a foundation to interpret the modal energy as a 'vibrational temperature' and the coupling power proportionality as a thermal law. SEA follows on from room acoustics and Sabine's formula and as such could be considered as a theory of statistical vibroacoustics [10] in the same way as exist statistical mechanics, statistical electromagnetism and so on.

Among numerous attempts to relax the more restrictive assumptions of SEA, several methods aim to predict the repartition of vibrational energy within subsystems where the field is not diffuse. But the behavior of local energy quantities is more subtle than it appears at first sight $[11,12,13]$ and it is generally not possible to obtain a closed set of equations on energy variables without introducing strong assumptions.

To circumvent this difficulty, it has been proposed by several authors [14, $15,16,17,18,19,20,21,22,23,24,25]$ to start from an integral representation of the vibrational field and to calculate a quadratic quantity by neglecting to various degrees the coherence between the boundary sources. When all sources are incoherent, it results in a boundary integral equation 
on energy that may be solved by a collocation or Galerkin method. Although they have been developed in various contexts, all these methods suggest more or less the idea that vibrational energy propagates in structures like radiant thermal energy. This thermal analogy is clearly not equivalent to the thermal conductivity proposed in other respects (see Refs. [26, 27, 23] for a discussion on this point). The radiative transfer analogy is more apparent in room acoustics with the so-called radiosity method [28, 29, 30, 31, 32]. Furthermore, when the vibrational field tends to be diffuse, it may be checked that the coupling power proportionality of statistical energy analysis is obtained as a limit case [33]. In this concern, let us also mention the recently proposed method called dynamical energy analysis which investigates the gap between full ray-tracing and statistical energy analysis [34, 35, 36].

The purpose of this paper is to review the theoretical framework of an extension of the radiosity method to specular reflection and diffraction which may serve as a basis for a generalization of statistical energy analysis. The outline of the paper is as follows. In Section 2 we introduce some generalities about representation of a vibration field in terms of radiant energy supplied by actual and fictitious sources. In Section 3 are derived the balance equations governing the emissive power of fictitious sources. The special case of diffuse reflection and the radiosity equation is introduced in Section 4. Two problems which enlarge the scope of classical radiosity are then discussed, specular reflection in Section 5 and diffraction in Section 6. The paper finishes with some concluding remarks.

\section{Energy representation of a wave field}

The laws of radiative energy transfer is based on two principles; the linear superposition principle and Huygens' principle.

If the vibrational field is sufficiently 'disordered' then we may neglect all correlations between rays and energy variables turn out to be well-suited to describe the field state. Two rays emanating from a single source with a slightly different direction take different paths and are subjected to different events such as reflection, diffraction, diffusion etc. before eventually reaches the same receiver point. They rapidly 'forget' their phase so that after travelling a long distance, their energies may be added signifying that interference effects can be neglected.

Huygens' principle states that each point of reflection, refraction, diffraction etc. behaves like a secondary source from which emanate spherical 

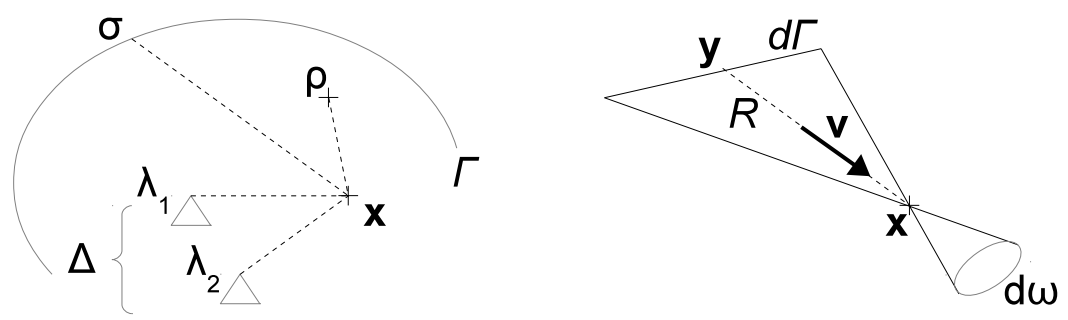

Figure 1: Actual and fictitious sources $\rho, \sigma$ and $\lambda$.

waves. A more modern form is the so-called Helmoltz-Kirchhoff formula or any representation formula by Green's function. The principle of all these formulas is that in the presence of an obstacle, the field is unchanged if one removes the obstacle and replaces it by appropriate fictitious sources. The field is therefore the sum of a direct field (the field that would be without the obstacle) and the field produced by fictitious sources.

Let $\Omega$ be a domain whose regular boundary is $\Gamma$ and whose set of diffracting points is $\Delta$. We admit that $\Omega$ may contain volume sources $\rho$. By virtue of Huygens' principle, we also put surface sources $\sigma$ on $\Gamma$ and diffraction sources $\lambda_{i}$ on $\Delta$ (Fig. 1). The energy density $W$ at any point $\mathbf{x}$ and time $t$ is given by (assuming incoherence),

$$
W(\mathbf{x}, t)=\int_{\Omega} \frac{\rho\left(\mathbf{y}, t^{\prime}\right)}{4 \pi c R^{2}} d \Omega_{\mathbf{y}}+\int_{\Gamma} \frac{\sigma\left(\mathbf{y}, \mathbf{v}, t^{\prime}\right)}{4 \pi c R^{2}} d \Gamma_{\mathbf{y}}+\sum_{i \in \Delta} \frac{\lambda_{i}\left(\mathbf{v}, t^{\prime}\right)}{4 \pi c R^{2}}
$$

where $R=|\mathbf{x}-\mathbf{y}|$ is the source-receiver distance, $\mathbf{v}=(\mathbf{x}-\mathbf{y}) / R$ the sourcereceiver direction, $t^{\prime}=t-R / c$ the retarded time and $c$ the sound speed (Fig. 1). The domain sources $\rho$ have been assumed omnidirectional but $\sigma$ and $\lambda_{i}$ depend on the emission direction $\mathbf{v}$.

Inside the domain, we may also introduce the specific radiative intensity $I(\mathbf{x}, \mathbf{v}, t)$ defined as the energy flow per unit solid angle and unit area normal to the rays. While the energy density $W$ only depends on the position $\mathbf{x}$ and the time $t$, the specific intensity depends in addition on the direction vector $\mathbf{v}$. Considering a position $\mathbf{x}$ and an infinitesimal solid angle $d \omega$ about a direction $\mathbf{v}$, the specific intensity is,

$$
I(\mathbf{x}, \mathbf{v}, t) d \omega=\int_{d \omega} \frac{\rho\left(\mathbf{y}, t^{\prime}\right)}{4 \pi R^{2}} d \Omega_{\mathbf{y}}+\frac{\sigma\left(\mathbf{y}, \mathbf{v}, t^{\prime}\right)}{4 \pi R^{2}} d \Gamma+\sum_{i} \frac{\lambda_{i}\left(\mathbf{v}, t^{\prime}\right)}{4 \pi R^{2}}
$$


where the integration is performed over the cone of vertex $\mathbf{x}$ and angle $d \omega$, $d \Gamma$ is the part of boundary seen from $\mathbf{x}$ into the solid angle $d \omega$ and the last sum is restricted to diffraction points lying in the cone (Fig. 1).

An equivalent representation is obtained for two-dimensional domains by substituting the kernel $1 / 4 \pi R^{2}$ with $1 / 2 \pi R$. This is useful for instance for wave propagation in structural components such as plates, membranes, shells and so on. But when the medium is dispersive, $c$ must be understood as the group velocity since this is the speed of energy flow.

The symbols $\rho, \sigma, \lambda$ may readily be interpreted in terms of energy flux. Consider a point source located at $\mathbf{x}_{0}$ or equivalently a source density $\rho(\mathbf{y}, t)=$ $\delta\left(\mathbf{y}-\mathbf{x}_{0}\right) \rho(t)$. The intensity vector is radial and has magnitude $\rho / 4 \pi R^{2}$ at a distance $R$. By calculating the flux of intensity over an infinitely small sphere of radius $\epsilon$, it yields,

$$
\lim _{\epsilon \rightarrow 0} \int_{4 \pi} \frac{\rho(t-\epsilon / c)}{4 \pi \epsilon^{2}} \epsilon^{2} d \omega=\rho(t)
$$

So, $\rho$ is the power density of domain sources and therefore has units $\mathrm{W} / \mathrm{m}^{3}$. We may assume that the power $\rho$ of domain sources is always known. These sources represent actions of external loads and therefore must be specified for a well-posed problem. External loadings are usually expressed in terms of force or moment applied to a structure or volume velocity in a fluid. They may be specified either by their root-mean-square value or power spectral density. In all cases, asymptotic relationships based on point-mobility of the infinite system are available in the literature to convert these values into a power $[37,38,39]$.

The specific intensity $I(\mathbf{x}, \mathbf{u}, t)$ leaving the surface in direction $\mathbf{u}$ is calculated by dividing the infinitesimal power into the solid angle $d \omega$ by the apparent normal surface $\cos \theta d \Gamma$ where $\theta$ is the polar angle with the normal to the boundary (Fig. 2). So for an infinitesimal surface $d \Gamma$ and an emission angle $\theta$,

$$
I(\mathbf{x}, \mathbf{u}, t) d \omega \cos \theta d \Gamma=\lim _{\epsilon \rightarrow 0} \frac{\sigma(\mathbf{x}, \mathbf{u}, t-\epsilon / c)}{4 \pi \epsilon^{2}} \epsilon^{2} d \omega d \Gamma
$$

The specific intensity leaving in direction $\mathbf{u}$ is therefore $\sigma(\mathbf{x}, \mathbf{u}, t) / 4 \pi \cos \theta$. A perfectly diffuse source has a constant specific intensity. In two-dimensional systems, the factor $4 \pi$ is replaced with $2 \pi$.

Alternatively $\sigma / 4 \pi$ is the power per unit area of surface and solid angle and is sometimes called the directional emitted flux or directional emissive power [40]. It has units of $\mathrm{W} / \mathrm{m}^{2}$. 


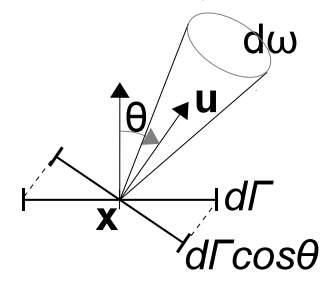

Figure 2: Specific radiative intensity from a surface source.

The radiosity $B$ is the total energy flow leaving per unit area of the surface. However some authors prefer to define the radiosity as the incident energy rate. The radiosity is obtained by integrating the specific intensity over all emission directions, $B=\int I \cos \theta d \omega$, giving

$$
B(\mathbf{x}, t)=\int_{2 \pi} \sigma(\mathbf{x}, \mathbf{u}, t) \frac{d \omega}{4 \pi}
$$

where the integration is performed over the half-sphere of inward directions.

\section{Radiative transfer equation}

A representation of an energy field like in Eq. (1) immediately raises the question of the determination of fictitious source powers $\sigma$ and $\lambda$.

Let the bidirectional reflectivity $[40] \mathcal{R}(\mathbf{v}, \mathbf{u})$ of the boundary be the ratio of radiative intensity $I(\mathbf{x}, \mathbf{u}, t)$ reflected in direction $\mathbf{u}$ with emission angle $\theta$ and the incident flux $I(\mathbf{x}, \mathbf{v}, t) \cos \varphi$ from direction $\mathbf{v}$ with incidence angle $\varphi$. The bidirectional reflectivity is also referred to as reflection matrix [41]. At a fixed point $\mathbf{x}$ of the boundary, the specific intensity $I(\mathbf{x}, \mathbf{u}, t)$ leaving in direction $\mathbf{u}$ is the sum of contributions from all incident directions $\mathbf{v}$ times $\mathcal{R}(\mathbf{v}, \mathbf{u})$. It yields,

$$
I(\mathbf{x}, \mathbf{u}, t)=\int \mathcal{R}(\mathbf{v}, \mathbf{u}) I(\mathbf{x}, \mathbf{v}, t) \cos \varphi d \omega
$$

This equation expresses conversion in phase space of energy flux at any point of the boundary. But at a boundary point $\mathbf{x}$, the specific radiative intensity 
is $I=\sigma / 4 \pi \cos \theta$ while the incident flux is given in Eq. (2). Substitution gives,

$$
\begin{aligned}
\frac{\sigma(\mathbf{x}, \mathbf{u}, t)}{\cos \theta}= & \int_{\Omega} \mathcal{R}(\mathbf{v}, \mathbf{u}) \rho\left(\mathbf{y}, t^{\prime}\right) \frac{\cos \varphi}{R^{2}} d \Omega_{\mathbf{y}}+\int_{\Gamma} \mathcal{R}(\mathbf{v}, \mathbf{u}) \sigma\left(\mathbf{y}, \mathbf{v}, t^{\prime}\right) \frac{\cos \varphi}{R^{2}} d \Gamma_{\mathbf{y}} \\
& +\sum_{i} \mathcal{R}(\mathbf{v}, \mathbf{u}) \lambda_{i}\left(\mathbf{v}, t^{\prime}\right) \frac{\cos \varphi}{R^{2}}
\end{aligned}
$$

This is an integral equation which specifies the unknown $\sigma$ at any position $\mathbf{x}$ and in any direction $\mathbf{u}$ as dependent on $\sigma$ at all other positions $\mathbf{y}$ and directions $\mathbf{v}$.

A similar method applies for $\lambda$. Let define the bidirectional diffractivity $\mathcal{D}(\mathbf{v}, \mathbf{u})$ by analogy with the bidirectional reflectivity as the ratio of the emitted power per unit solid angle about $\mathbf{u}$ and the incident radiative intensity from direction $\mathbf{v}$. The total power emitted is the sum of contributions from all incident directions,

$$
\frac{\lambda_{i}(\mathbf{u}, t)}{4 \pi}=\int \mathcal{D}(\mathbf{v}, \mathbf{u}) I(\mathbf{x}, \mathbf{v}, t) d \omega
$$

By substituting Eq. (2),

$$
\begin{aligned}
\lambda_{i}(\mathbf{u}, t)= & \int_{\Omega} \mathcal{D}(\mathbf{v}, \mathbf{u}) \rho\left(\mathbf{y}, t^{\prime}\right) \frac{d \Omega_{\mathbf{y}}}{R^{2}}+\int_{\Gamma} \mathcal{D}(\mathbf{v}, \mathbf{u}) \sigma\left(\mathbf{y}, \mathbf{v}, t^{\prime}\right) \frac{d \Gamma_{\mathbf{y}}}{R^{2}} \\
& +\sum_{j \neq i} \mathcal{D}(\mathbf{v}, \mathbf{u}) \lambda_{j}\left(\mathbf{v}, t^{\prime}\right) \frac{1}{R^{2}}
\end{aligned}
$$

Again, the unknowns $\lambda_{i}$ are determined by an integral equation where both $\lambda_{j}$ and $\sigma$ are involved.

\section{Radiosity method}

The radiosity method has been introduced in acoustics by Kuttruff in Ref. [28, 29]. Originally developed in thermics to compute the temperature of facing walls in thermal equilibrium by the so-called standard method or view factor method, this is a popular method in optics for illumination problems. The radiosity method may be readily transposed in acoustics provided that a finite sound speed is introduced. The advantage is that it provides a fruitful piece of information for transient regimes. 
The radiosity method applies to rooms with perfect diffusely reflecting walls. Diffraction is usually not taken into account so that we set $\lambda_{i}=0$ in Eqs. $(1,7)$. The reflectivity of a perfectly diffusing surface is the constant $[41]$

$$
\mathcal{R}(\mathbf{v}, \mathbf{u})=\frac{1-\alpha}{\pi}
$$

where $\alpha$ is the absorptivity of the wall. Most often, we may consider that the absorptivity does not depend on the incidence angle. Furthermore, a diffuse reflection follows Lambert's law which states that the specific intensity of a diffuse source does not depend on the emission direction. The brightness of a diffuse surface is the same whatever the observation angle. The Lambert law or cosine law is,

$$
\frac{\sigma(\mathbf{x}, \mathbf{u}, t)}{4 \pi \cos \theta}=K
$$

By a direct integration over all emission directions and a comparison with Eq. (14), it immediately follows that the constant $K$ equals $B / \pi$. Thus, by substituting Lambert's law (11) and the diffuse reflectivity (10) into Eq. (7), we obtain the radiosity equation,

$$
B(\mathbf{x}, t)=(1-\alpha(\mathbf{x}))\left[E(\mathbf{x}, t)+\int_{\Gamma} B\left(\mathbf{y}, t-\frac{R}{c}\right) \frac{\cos \varphi \cos \theta}{\pi R^{2}} d \Gamma_{\mathbf{y}}\right]
$$

where $E(\mathbf{x}, t)$ is the contribution of the direct field,

$$
E(\mathbf{x}, t)=\int_{\Omega} \rho\left(\mathbf{y}, t-\frac{R}{c}\right) \frac{\cos \varphi}{4 \pi R^{2}} d \Omega_{\mathbf{y}}
$$

In the two above equations, $\varphi$ is the incidence angle at $\mathbf{y}, \theta$ the emission angle at $\mathbf{y}$ and $R=|\mathbf{x}-\mathbf{y}|$ the source receiver distance.

The most important consequence of the radiosity equation is that it allows to determine the sound decay after sources have been switched off even when the sound field is not diffuse. The radiosity equation turns out to be therefore an important generalization of Sabine's theory to predict the reverberation time in rooms of arbitrary shape and absorption factors. Miles [42] observed that after a rapid transient state, the sound ultimately decays exponentially at the same rate at each location in the enclosure. By introducing an exponential time dependence $B(\mathbf{y}, t)=B(\mathbf{y}) \exp (-\lambda t)$ in Eq. (12) with $E=0$, it yields,

$$
B(\mathbf{x})=(1-\alpha(\mathbf{x}))\left[\int_{\Gamma} B(\mathbf{y}) \exp \left(\lambda \frac{R}{c}\right) \frac{\cos \varphi \cos \theta}{\pi R^{2}} d \Gamma_{\mathbf{y}}\right]
$$




\begin{tabular}{|c||c|c|c||c|c|}
\hline absorption & Sabine & Eyring & Millington & Gilbert & Kuttruff \\
\hline \hline uniform & 28.7 & 28.5 & 28.5 & 29.5 & 29.5 \\
\hline floor $\alpha=0.8$ & 0.99 & 0.84 & 0.50 & 0.80 & 0.79 \\
\hline wall $\alpha=0.8$ & 5.08 & 4.93 & 2.75 & 6.78 & 6.96 \\
\hline
\end{tabular}

Table 1: Reverberation time (in s) for a rectangular room of size $20 \times 30 \times 5 \mathrm{~m}^{3}$ by standard formula (Sabine, Eyring, Millington) and the radiosity equation (14) by two algorithms (Gilbert [45], Kuttruff [30])

The calculation of reverberation time then reduces to the solving of the above equation in $\lambda$. Several algorithms and closed-form solutions have been proposed in the literature $[43,44,45,46,30,31]$.

An example is proposed in Table 1 . The room is rectangular $20 \times 30 \times 5 \mathrm{~m}^{3}$ and has volume $V=3000 \mathrm{~m}^{3}$, surface area $S=1700 \mathrm{~m}^{2}$ and a uniform absorption $\alpha=0.01$ excepted in one side where a very high absorption $\alpha=0.8$ is prescribed. The reverberation time $T_{r}$ is estimated by the three standard formula Sabine, Eyring and Millington and by Eq. (14) with $T_{r}=13.8 / \lambda$. In the uniform case $(\alpha=0.01$ on all surfaces) the sound field is diffuse. All methods give more or less the same result. When the floor $20 \times 30$ is highly absorbing, a strong difference appears between Sabine, Eyring and Millington formula since the difference between $\alpha$ and $-\ln (1-\alpha)$ is large for $\alpha=0.8$. But, the radiosity equation gives a result of $0.8 \mathrm{~s}$ which is significantly less than Sabine's result and close to Eyring's result. Finally, for a highly absorbing wall $(10 \times 5)$ the trend is reversed and all standard formula predict a reverberation time lower than the one of radiosity.

Another important application of the radiosity method is that it provides an alternative method to ray-tracing for the computation of sound pressure level maps in rooms with stationary sound sources. The formal proof of equivalence with ray-tracing and some numerical examples are given in Ref. [47, 48].

\section{Specular reflection}

The most intriguing property of the representation (1) is that it accounts for specular reflection [49]. To illustrate it, let us consider the simple situation of a steady-state point source in front of a perfect infinite mirror (Fig. 3). In 


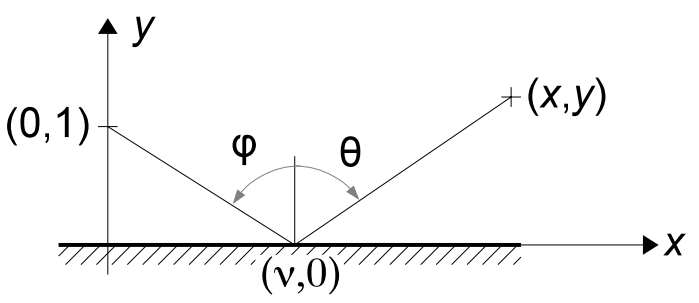

Figure 3: Point source facing a perfect mirror. The source is at $(0,1)$, the receiver at $(x, y)$ and the reflecting point at $(\nu, 0)$. The incidence angle is $\varphi$ and the emission angle $\theta$.

a two-dimensional space, the direct field is,

$$
W_{d}(x, y)=\frac{1}{2 \pi c \sqrt{x^{2}+(y-1)^{2}}}
$$

if the source is at $x=0$ and $y=1$ and has a unit power. If furthermore the reflecting line is $y=0$ then we set a fictitious layer $\sigma(\nu, \theta)$ where $\nu$ is the abscissa and $\theta$ the emission angle.

In the right hand-side of Eq. (7), the source power $\rho=\delta(x) \delta(y-1)$ is a delta Dirac function and the first integral reduces to $\mathcal{R}(\varphi, \theta) \cos \varphi / R$. But for a perfect mirror, the specular bidirectional reflectivity is [41],

$$
\mathcal{R}(\varphi, \theta)=\frac{\delta(\theta-\varphi)}{\cos \varphi}
$$

Furthermore, the distance from the point source to the boundary point $x=\nu$, $y=0$ is $R=\sqrt{\nu^{2}+1}$ and the incidence angle is $\varphi=\arctan \nu$. Substituting in Eq. (7) gives,

$$
\sigma(\nu, \theta)=\delta(\theta-\arctan \nu) \frac{\cos \theta}{\sqrt{\nu^{2}+1}}
$$

The reflected field is given by the second integral of Eq. (1). At a boundary point $\nu$ the emission angle in view of the receiver point $x, y$ is $\theta=\arctan (x-\nu) / y$ and satisfies $\cos \theta=y / \sqrt{(x-\nu)^{2}+y^{2}}$. The reflected field is therefore,

$$
W_{r}(x, y)=\int_{-\infty}^{\infty} \delta\left(\arctan \frac{x-\nu}{y}-\arctan \nu\right) \frac{\cos \theta}{\sqrt{\nu^{2}+1}} \frac{d \nu}{2 \pi c \sqrt{(x-\nu)^{2}+y^{2}}}
$$


After simplification,

$$
W_{r}(x, y)=\frac{1}{2 \pi c \sqrt{x^{2}+(y+1)^{2}}}
$$

So, the reflected field shrinks to a single image-source located at $x=0$, $y=-1$. Of course, this is a well-known property of rays. But the fact that the formation of image-sources is representable by an integral of type $\int \sigma / 2 \pi c R d \nu$ points out that Eq. (1) accounts for a wide class of phenomena in spite of the fact that interferences have been neglected.

The extension of the radiosity method to specular reflection has been studied for a long time in thermics $[50,51,40]$ and more recently in acoustics [52]. The classical solution is based on the image-source technique and the introduction of specular view-factors. But some non-standard solutions have also been proposed recently $[15,53]$. In statistical energy analysis, the image-source technique also provides a powerful extension of the method when the sound field is not diffuse in the enclosure [54].

\section{Multiple diffraction}

Another interesting example of problems to which the representation equation (1) may give an elegant solution is that of multiple diffraction $[55,56]$. To illustrate the problem, let us consider a two-dimensional acoustic medium with an incident stationary plane wave on two small obstacles localized respectively at the origin and $(0, L)$ as shown in Fig. 4.

To solve this problem, two fictitious sources $\lambda_{1}$ and $\lambda_{2}$ are set to the diffraction points. Their directional emissive powers $\lambda_{i} / 2 \pi$ are given by Eq. (9) where the source term is $2 \pi I_{0}$ and no source $\sigma$ has been placed. Then

$$
\begin{aligned}
& \lambda_{1}(\theta)=\mathcal{D}(\varphi, \theta) 2 \pi I_{0}+\mathcal{D}(0, \theta) \frac{\lambda_{2}(\pi)}{L} \\
& \lambda_{2}(\theta)=\mathcal{D}(\varphi, \theta) 2 \pi I_{0}+\mathcal{D}(\pi, \theta) \frac{\lambda_{1}(0)}{L}
\end{aligned}
$$

Eqs. (20), (21) give the emissive powers $\lambda_{1}, \lambda_{2}$ in any direction provided that they are known in grazing directions. We must first determine $\lambda_{1}(0)$ and $\lambda_{2}(\pi)$. Applying the two above equations respectively to $\theta=0$ and $\theta=\pi$ 


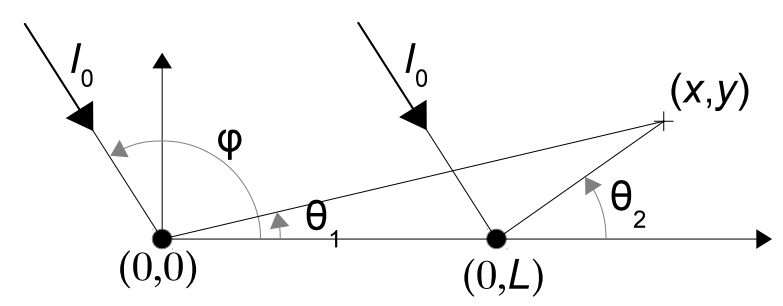

Figure 4: Double diffraction. The source is at infinite with incidence $\varphi$, the receiver at $(x, y)$ and the diffracting points at $(0,0)$ and $(0, L)$. The emission angle is $\theta$.

gives,

$$
\begin{aligned}
& \lambda_{1}(0)=\mathcal{D}(\varphi, 0) 2 \pi I_{0}+\mathcal{D}(0,0) \frac{\lambda_{2}(\pi)}{L} \\
& \lambda_{2}(\pi)=\mathcal{D}(\varphi, \pi) 2 \pi I_{0}+\mathcal{D}(\pi, \pi) \frac{\lambda_{1}(0)}{L}
\end{aligned}
$$

This set of linear equations is readily solved, giving

$$
\begin{aligned}
& \lambda_{1}(0)=2 \pi I_{0} \frac{\mathcal{D}(\varphi, 0)+\mathcal{D}(0,0) \mathcal{D}(\varphi, \pi) / L}{1-\mathcal{D}(0,0) \mathcal{D}(\pi, \pi) / L^{2}} \\
& \lambda_{2}(\pi)=2 \pi I_{0} \frac{\mathcal{D}(\varphi, \pi)+\mathcal{D}(\pi, \pi) \mathcal{D}(\varphi, 0) / L}{1-\mathcal{D}(0,0) \mathcal{D}(\pi, \pi) / L^{2}}
\end{aligned}
$$

Finally, the energy density at any point is found by applying Eq. (1),

$$
W(x, y)=\frac{I_{0}}{c}+\frac{\lambda_{1}\left(\theta_{1}\right)}{2 \pi c \sqrt{x^{2}+y^{2}}}+\frac{\lambda_{2}\left(\theta_{2}\right)}{2 \pi c \sqrt{(x-L)^{2}+y^{2}}}
$$

This is the solution to the problem of double diffraction.

\section{Transition to statistical energy analysis}

When the field becomes diffuse in a domain, a simple law emerges: The energy leaving the system through the boundary is proportional to the total energy,

$$
P_{1 \rightarrow 2}=\eta_{12} \omega E_{1}
$$


where $E_{1}$ is the total energy, $P_{1 \rightarrow 2}$ the outgoing power, $\omega$ the frequency $(\mathrm{rad} / \mathrm{s})$ and $\eta_{12}$ the so-called coupling loss factor. When two subsytems are connected by a common boundary, the net exchanged energy may be decomposed as a difference of two terms $P_{12}=P_{1 \rightarrow 2}-P_{2 \rightarrow 1}=\omega\left(\eta_{12} E_{1}-\eta_{21} \omega E_{2}\right)$. This is the coupling power proportionality law well-known in statistical energy analysis.

This phenomenon is correctly predicted by the radiative transfer equations. Assuming for convenience a unique stationary point source located at $\mathbf{x}_{0}$ and no diffracting point, the total energy is obtained by integrating the energy density $W(\mathbf{x})$ of Eq. (1) over the domain,

$$
E_{1}=\int_{\Omega} \frac{d \Omega_{\mathbf{x}}}{4 \pi c R_{0}^{2}}+\int_{\Omega} \int_{\Gamma} \frac{\sigma(\mathbf{y}, \mathbf{v})}{4 \pi c R^{2}} d \Gamma_{\mathbf{y}} d \Omega_{\mathbf{x}}
$$

where $R_{0}=\left|\mathbf{x}_{0}-\mathbf{x}\right|$ is the source-receiver distance and $R=|\mathbf{x}-\mathbf{y}|$. The outgoing power is the flux of the normal component of the incident radiative intensity $I(\mathbf{x}, \mathbf{v}) \cos \varphi$,

$$
P_{1 \rightarrow 2}=\alpha\left(\int_{\Gamma} \frac{\cos \varphi}{4 \pi R_{0}^{2}} d \Gamma_{\mathbf{x}}+\int_{\Gamma} \int_{\Gamma} \sigma(\mathbf{y}, \mathbf{v}) \frac{\cos \varphi}{4 \pi R^{2}} d \Gamma_{\mathbf{y}} d \Gamma_{\mathbf{x}}\right)
$$

Let us consider the canonical problem of a system of volume $V$ lightly damped with reflecting boundaries excepted a small aperture of area $S$ of totally absorbing boundary. We may wonder if the ratio $P_{1 \rightarrow 2} / E_{1}$ tends to the asymptotic limit,

$$
\eta_{12} \omega=\frac{S c}{4 \pi V} \alpha
$$

predicted by statistical energy analysis.

In Table 2 is shown the evolution of $P_{1 \rightarrow 2} / E_{1}$ and $\eta_{12} \omega$ versus $S$ for a room of $20 \times 30 \times 5 \mathrm{~m}^{3}$ with an aperture centred on a wall $30 \times 5$. The aperture has $\alpha=0.99$ while $\alpha=0$ for other surfaces. A single point source of $1 \mathrm{~W}$ is located at the centre of the room. To perform the calculation, the radiosity equation (12) has been solved in $B(\mathbf{y})$ in steady-state condition by a collocation method with a mesh of about 1200 triangles. $E_{1}$ and $P_{1 \rightarrow 2}$ have been calculated by Eqs. $(28,29)$ where $\sigma(\mathbf{y}, \mathbf{v})=4 B(\mathbf{y}) \cos \theta$ have been substituted. The number of receiver points to assess $E_{1}$ is 36 . The ratio of standard deviation and mean value of energies also appears in Table 2 . The results clearly indicates that a large discrepancy between $\eta_{12}$ and $P_{1 \rightarrow 2} / E_{1}$ is observed for large apertures where the direct field is dominant 


\begin{tabular}{|c||c|c|c|c|c|}
\hline aperture $\left(\mathrm{m}^{2}\right)$ & $28 \times 4$ & $10 \times 4$ & $4 \times 4$ & $2 \times 2$ & $1 \times 1$ \\
\hline \hline$\eta_{12} \omega\left(\mathrm{s}^{-1}\right)$ & 3.1416 & 1.1220 & 0.4488 & 0.1122 & 0.0280 \\
\hline$P_{1 \rightarrow 2} / E_{1}\left(\mathrm{~s}^{-1}\right)$ & 1.8220 & 0.8624 & 0.3942 & 0.1094 & 0.0282 \\
\hline std/mean $(\%)$ & 126 & 58.8 & 26.9 & 7.45 & 1.91 \\
\hline
\end{tabular}

Table 2: Comparison of the empirical coupling loss factor $P_{1 \rightarrow 2} / E_{1}$ with the asymptotic limit $\eta_{12} \omega$ of statistical energy analysis for a room of size $20 \times 30 \times 5 \mathrm{~m}^{3}$ with perfectly reflecting $(\alpha=0)$ walls, floor and ceiling and an aperture $(\alpha=0.99)$ centred on the wall $30 \times 5$. The point source is centred in the room.

(large std/mean). But the convergence is good for small apertures when the field becomes diffuse inside the room (small std/mean).

\section{Conclusion}

In this study, the theoretical framework for a generalization of the radiosity method in acoustics and statistical energy analysis in vibro-acoustics has been presented in detail. The main features are the followings. The vibrational field, described in terms of energy density and specific intensity, is constructed as a linear superposition of actual and fictitious sources positioned in the domain, on the boundary and at the diffraction points. The emissive power of these sources is driven by the balance of radiant exchanges as in optics or thermics.

The method whose principle is well-known in thermics seems to be also useful in vibro-acoustics. The development of the radiosity method in acoustics and its application to sound illumination problem in room acoustics or reverberation time determination beyond the validity of Sabine's law underline its importance in engineering. Furthermore, its extension to specular reflection and diffraction highlights that it may be also useful in a larger class of problems, including sound radiation, structural response, sound transmission [57]. From this point of view, radiative exchange equations look like a natural extension of statistical energy analysis when energy fields are not diffuse.

\section{Acknowledgements}

The authors sincerely acknowledge the Labex Celya (Lyon acoustic centre) in the framework of which this work was conducted. 


\section{References}

[1] P.J. Shorter, Modeling noise and vibration transmission in complex systems, in IUTAM Symposium on the Vibration Analysis of Structures with Uncertainties, IUTAM Bookseries 27 (2011) 141-156.

[2] R.H. Lyon, Statistical Energy Analysis of Dynamical Systems: Theory and Application, MIT Press, Massachusetts, Cambridge, 1975.

[3] R.H. Lyon, G. Maidanik, Power flow between linearly coupled oscillators, J. Acoust. Soc. Am. 34 (1962) 623-639.

[4] P. W. Smith, Response and radiation of structural modes excited by sound, J. Acoust. Soc. Am. 34 (1962) 640-647.

[5] D.E. Newland, Calculation of power flow between coupled oscillators, J. Sound Vib. 3 (1966) 262-276.

[6] B.R. Mace, L. Ji, The statistical energy analysis of coupled sets of oscillators, Proc. R. Soc. A 463 (2007) 1359-1377.

[7] T. Lafont, N. Totaro, A. Le Bot, Review of statistical energy analysis hypothesis in vibroacoustics, Proc. R. Soc. A 470 (2013) 20130515.

[8] L. Maxit and J.L. Guyader, Extension of SEA model to subsystems with non-uniform modal energy distribution, J. Sound Vib. 265(2) (2003) 337-358.

[9] N. Totaro, J.L. Guyader, Extension of the statistical modal energy distribution analysis for estimating energy density in coupled subsystems, J. Sound Vib. 331(13) (2012) 3114-3129.

[10] A. Le Bot, A. Carcaterra, D. Mazuyer, Statistical vibroacoustics and entropy concept, Entropy 12 (2010) 2418-2435.

[11] A. Carcaterra and A. Sestieri, Energy density equations and power flow in structures, J. Sound Vib. 188(2) (1995) 269-282.

[12] J.F. Li, J.C. Pascal and C. Carle, Energy fields of partially coherent sources, J. Acoust. Soc. Am. 103(2) (1998) 962-972. 
[13] A. Le Bot, Geometric diffusion of vibrational energy and comparison with the vibrational conductivity approach, J. Sound Vib. 212(4) (1998) 637-647.

[14] A. Le Bot, A vibroacoustic model for high frequency analysis, J. Sound Vib. 211(4) (1998) 537-554.

[15] L.P. Franzoni, D.B. Bliss and J.W. House, An acoustic boundary element method based on energy and intensity variables for prediction of high frequency broadband sound fields, J. Acoust. Soc. Am., 110 (2001) 3071-3080.

[16] V. Cotoni and A. Le Bot, Radiation of plane structures at high frequency using an energy method, International Journal of Acoustics and Vibration 6(4) (2001) 209-214.

[17] A. Le Bot, Energy transfer for high frequencies in built-up structures, J. Sound Vib. 250(2) (2002) 247-275.

[18] J.K. Kim and J.G. Ih, Prediction of sound level at high-frequency bands by means of a simplified boundary element method, J. Acoust. Soc. Am. 112(6) (2002) 2645-2655.

[19] E. Sarradj, High frequency boundary integral method as an alternative to statistical energy analysis, in: 10th ICSV, Stockholm, Sweden, 2003.

[20] J.L. Guyader, Integral equation for frequency averaged quadratic pressure, Acta Acustica united with Acustica 90(2) (2004) 232-245.

[21] D.W. Herrin, T.W. Wu and A.F. Seybert, The energy source simulation method, J. Sound Vib. 278 (2004) 135-153.

[22] A. Wang, N. Vlahopoulos, K. Wu, Development of an energy boundary element formulation for computing high-frequency sound radiation from incoherent intensity boundary conditions, J. Sound Vib. 278 (2004) 413436.

[23] A. Le Bot, Comparison of vibrational conductivity and radiative energy transfer methods, J. Sound Vib. 283 (2005) 135-151. 
[24] E. Sadoulet-Reboul, A. Le Bot, J. Perret-Liaudet, A hybrid method for vibroacoustics based on the radiative energy transfer method, J. Sound Vib. 303 (2007) 675-690.

[25] A. Pratellesi, M. Viktorovitch, N. Baldanzini et al., A hybrid formulation for mid-frequency analysis of assembled structures, J. Sound Vib. 309(35) (2008) 545-568.

[26] R.S. Langley, On the vibrational conductivity approach to high frequency dynamics for two-dimensional structural components, J. Sound Vib. 182 (1995) 637-657.

[27] A. Sestieri, A. Carcaterra, Space average and wave interference in vibration conductivity, J. Sound Vib. 263 (2003) 475-491.

[28] H. Kuttruff, Simulierte Nachhallkurven in Rechteckräumen mit diffusem Schallfeld, Acustica, 25 (1971) 333-342.

[29] H. Kuttruff, Room acoustics, 3rd ed., Elsevier Applied Science, London, 1991.

[30] H. Kuttruff, Energetic sound propagation in rooms, Acustica with Acta Acustica 83, (1997) 622-628.

[31] E.M. Nosal and M. Hodgson, Investigation of the validity of radiosity for sound-field prediction in cubic rooms, J. Acoust. Soc. Am. 116(6) (2004) 3505-3514.

[32] H. Zhang, Relaxation of sound fields in rooms of diffusely reflecting boundaries and its application in acoustical radiosity simulation, J. Acoust. Soc. Am. 119 (2006) 2189-2200.

[33] A. Le Bot, Derivation of statistical energy analysis from radiative exchanges, J. Sound Vib. 300 (2007) 763-779.

[34] G. Tanner, Dynamical energy analysisDetermining wave energy distributions in vibro-acoustical structures in the high-frequency regime, J. Sound Vib. 320 (2009) 1023-1038.

[35] D. Chappell, S. Giani, Tanner G., Dynamical energy analysis for builtup acoustic systems at high frequencies, J. Acoust. Soc. Am. 130(3) (2011) 1420-1429. 
[36] D. Chappell, Tanner G., S. Giani, Boundary element dynamical energy analysis: A versatile method for solving two or three dimensional wave problems in the high frequency limit, Journal of Computational Physics 231(18) (2012) 6181-6191.

[37] E.J. Skudrzyk, Vibrations of a system with a finite or an infinite number of resonances, J. Acoust. Soc. Am. 30 (1958) 1140-1152.

[38] E.J. Skudrzyk, Sound radiation of a system with a finite or an infinite number of resonances, J. Acoust. Soc. Am. 30 (1958) 1152-1158.

[39] H.G.D. Goyder and R.G. White, Vibrational power flow machines into built-up structures,part I: Introduction and approximate analyses of of beam and plate-like foundations, J. Sound Vib. 68 (1980) 77-96.

[40] M. F. Modest, Radiative heat transfer, (Mc Graw-Hill), 1993.

[41] W.B. Joyce, Exact effect of surface roughness on the reverberation time of a uniformly absorbing spherical enclosure, J. Acoust. Soc. Am. 64 (1978) 1429-1436.

[42] R. Miles, Sound field in a rectangular enclosure with diffusely reflecting boundaries, J. Sound Vib. 92 (1984) 203-226.

[43] R. Gerlach and V. Mellert, Der nachhallvorgang als markoffsche kette theorie und erste experimentelle überprüfung, Acustica 32 (1975) 211227.

[44] M. Carroll and C. Chien, Decay of reverberent sound in a spherical enclosure, J. Acoust. Soc. Am. 62 (1977) 1442-1446.

[45] E.N. Gilbert, An interative calculation of auditorium reverberation, J. Acoust. Soc. Am. 69 (1981) 178-184.

[46] H. Kuttruff, A simple iteration scheme for the computation of decay constants in enclosures with diffusely reflecting boundaries, J. Acoust. Soc. Am. 98(1) (1995) 288-293.

[47] A. Le Bot and A. Bocquillet, Comparison of an integral equation on energy and the ray-tracing technique for room acoustics, J. Acoust. Soc. Am. 108 (2000) 1732-1740. 
[48] R.T. Muehleisen, C.W. Beamer, Steady state acoustic radiosity for the prediction of sound pressure levels in enclosures with diffuse surfaces, Noise Control Engineering Journal 57(3) (2009) 244-262.

[49] A. Le Bot, A functional equation for the specular reflection of rays, J. Acoust. Soc. Am. 112 (2002) 1276-1287.

[50] E.R. Eckert and E.M. Sparrow, Radiative heat exchange between surfaces with specular reflection, Int. Journal of Heat Mass Transfer 3 (1961) 42-54.

[51] E.M. Sparrow, E.R. Eckert and V.K. Jonsson, An enclosure theory for radiative exchange between specularly and diffusely reflecting surfaces, Transactions of ASME, Journal of heat transfer (1962) 294-300.

[52] H. Kuttruff, Stationary propagation of sound energy in flat enclosures with partially diffuse surface reflection, Acustica with Acta Acustica 86(6) (2000) 1028-1033.

[53] V. Cotoni and A. Le Bot, Specular and diffuse reflections of rays in coupled thin plates at high frequencies, J. Sound Vib., 265 (2003) 2341.

[54] F. Sgard, H. Nelisse, N. Atalla, Prediction of the acoustical performance of enclosures using a hybrid statistical energy analysis: Image source model, J. Acoust. Soc. Am. 127(2) (2010) 784-795.

[55] E. Reboul, A. Le Bot and J. Perret-Liaudet, Introduction of acoustical diffraction in the radiative transfer method, Comptes Rendus Mécanique, 332(7) (2004) 505-511.

[56] E. Reboul, A. Le Bot and J. Perret-Liaudet, Radiative transfer equation for multiple diffraction, J. Acoust. Soc. Am. 118(3) (2005) 1326-1334.

[57] A. Le Bot, Energy exchange in uncorrelated ray fields of vibroacoustics, J. Acoust. Soc. Am. 120(3) (2006) 1194-1208. 